\section{RMD Open}

Rheumatic \&

Musculoskeletal Diseases

\title{
Cigarette smoking patterns preceding primary Sjögren's syndrome
}

\author{
Johannes Mofors (D), ${ }^{1}$ Albin Björk, ${ }^{1}$ Elina Richardsdotter Andersson, ${ }^{1}$ \\ Marika Kvarnström, ${ }^{1}$ Helena Forsblad d'Elia, ${ }^{2}$ Sara Magnusson-Bucher, ${ }^{3}$ \\ Leonid Padyukov, ${ }^{1}$ Ingrid Kockum, ${ }^{4}$ Jan Hillert, ${ }^{4}$ Per Eriksson, ${ }^{5}$ Thomas Mandl, ${ }^{6}$ \\ Gunnel Nordmark (D), ${ }^{7}$ Lars Alfredsson, ${ }^{4}$ Marie Wahren-Herlenius (D) 1,8
}

To cite: Mofors J, Björk A Richardsdotter Andersson E, et al. Cigarette smoking patterns preceding primary Sjögren's syndrome. RMD Open 2020;6: e001402. doi:10.1136/ rmdopen-2020-001402

- Supplemental material is published online only. To view please visit the journal online (http://dx.doi.org/10.1136/rmdo pen-2020-001402)

$\mathrm{JM}$ and $\mathrm{AB}$ contributed equally to this work.

Received 17 July 2020 Revised 20 August 2020 Accepted 9 September 2020

Check for updates

(c) Author(s) (or their employer(s)) 2020. Re-use permitted under CC BY-NC. No commercial re-use. See rights and permissions. Published by BMJ.

For numbered affiliations see end of article.

Correspondence to Marie Wahren-Herlenius marie.wahren@ki.se

\section{ABSTRACT}

Background Cigarette smoking is a well-established risk factor for several autoimmune diseases, but its role in primary Sjögren's syndrome (pSS) remains unclear. Here, we investigated the association between cigarette smoking and subsequent development of pSS.

Methods Information on smoking habits was collected from lifestyle habit questionnaires of patients with pSS $(n=815)$ and a matched control group $(n=4425)$ for a casecontrol study. Differences in smoking exposure were analysed by conditional logistic regression. Potential interactions between smoking and risk-associated human leucocyte antigens (HLA) were assessed by multivariate regression.

Results The fraction of patients with pSS having ever smoked prior to diagnosis was lower than in controls $(\mathrm{OR}$ $0.67,95 \% \mathrm{Cl} 0.55$ to 0.81 ). Current smoking at diagnosis was also less prevalent in cases (OR $0.37,95 \% \mathrm{Cl} 0.26$ to 0.53). However, period prevalence of smoking during early adulthood was not statistically different from controls (OR $0.89,95 \% \mathrm{Cl} 0.66$ to 1.22 ) but markedly decreased over time. This was partly due to patients being more prone to stop smoking, starting already 30 years prior to diagnosis (OR 2.01, 95\% $\mathrm{Cl} 1.22$ to 3.30). Smoking patterns were also stratified by autoantibody status, yielding similar estimates No interaction effects between HLA-DRB1 haplotypes and smoking were observed.

Conclusion The observed smoking patterns indicate that individuals who develop pSS smoke equally much as the general population during early life but are then more prone to stop. The data can be interpreted as smoking conferring protective effects, or reflecting early symptoms of pSS that affect smoking habits, emphasising the slow, progressive development of the disease.

\section{INTRODUCTION}

Primary Sjögren's syndrome (pSS) is a systemic autoimmune disease of multifactorial origin. ${ }^{12}$ Tissue-specific inflammation of exocrine glands, primarily salivary and lacrimal glands, is a hallmark of the disease and results in dryness of the mouth and eyes. Exocrine dysfunction of mucosal epithelium including that of the nasal cavity, oropharynx and bronchi is

\section{Key messages}

What is already known about this subject?

- Exposure to cigarette smoke is a well-established risk factor for several autoimmune diseases. However, the role of smoking in the aetiopathogenesis of pSS has not been thoroughly investigated and lacks consistent findings.

What does this study add?

- Overall, smoking does not appear to increase the risk of developing pSS.

- Novel observations indicate that individuals who are later diagnosed with pSS are more prone to discontinue smoking already several decades prior to diagnosis compared to the general population.

- There appears to be no interaction effects between smoking and risk-associated human leucocyte antigens (HLA) haplotypes in pSS.

How might this impact on clinical practice?

- Our findings indicate that disease progression may start several decennia prior to becoming clinically overt, highlighting potential benefits from earlier detection and treatment of the condition.

common. A majority of patients also present with persistent fatigue and arthralgia, and subsets of patients develop systemic, organ-specific manifestations in, for example, the haematological, cutaneous, renal or neurological systems. ${ }^{3}{ }^{4}$ The presence of autoantibodies in serum targeting the Ro/SSA and La/SSB antigens demarks a patient subset with distinct major histocompatibility complex genotypes, whereas genetic features in patients with pSS without these autoantibodies have not been demonstrated to significantly differ from that of controls. ${ }^{5-8}$ Treatment of pSS is mainly symptomatic since evidence-based treatment with a significant impact on the disease course is currently lacking. ${ }^{3}$ Also, knowledge on factors suitable for preventive measures is insufficient. $^{9}$ 
The precise nature of why pSS occurs remains elusive, but disease processes are thought to be initiated through a complex interaction between genetic and environmental factors. Reliable data on monozygotic twin concordance rates for pSS are lacking, but familial aggregation of the disease has been reported. ${ }^{10}$ Data from other systemic autoimmune diseases indicate concordance rates of about $3-8 \%$ in dizygotic twins and $12-15 \%$ in monozygotic twins, ${ }^{11-13}$ revealing that environmental factors most likely have a significant contribution to disease risk.

The most significant genetic associations with pSS are found within the human leucocyte antigen (HLA) locus. ${ }^{14} 15$ This is of particular relevance since specific HLA haplotypes may interact with environmental factors such as smoking to increase the risk of seropositive rheumatoid arthritis (RA) and multiple sclerosis (MS) ${ }^{16} 17$ Notably, the strong HLA class II association with pSS is only found in autoantibody-positive disease, ${ }^{8}$ and specifically, HLA-DRB $1 * 03$ is associated with production of both anti-Ro/SSA and anti-La/SSB autoantibodies, while HLA-DRB1*15 is associated with anti-Ro/SSA only. ${ }^{5}$

Infections have repeatedly been proposed as an environmental risk factor for $\mathrm{pSS},{ }^{18-20}$ but the roles of other environmental factors remain to be determined. Cigarette smoking, which is a well-established risk factor in autoimmune diseases such as RA and $\mathrm{MS},{ }^{21} 22$ has not been thoroughly studied in pSS and reports present diverging data. ${ }^{23-27}$ Further, the relevance of dose of smoking in pack-years and potential gene-environment interactions have not been assessed.

To better define the influence of cigarette smoking on the risk of developing pSS, we performed the present case-control study using a well-characterised and large Swedish cohort. We quantified the levels of smoking exposure and stratified disease subsets based on the presence or absence of autoantibodies and investigated potential interactions between smoking and HLAs associated with rheumatic disease.

\section{MATERIALS AND METHODS}

\section{Study population and study design}

Patient data in this report were collected within the project Genes and Environment in Sjögren's Syndrome (GESS), which is a study comprising prevalent pSS cases in Sweden. Patients fulfiling the AmericanEuropean Consensus Group criteria $^{28}$ for pSS $(n=815)$ diagnosed at the Departments of Rheumatology at the University Hospitals in Gothenburg, Malmö/Lund, Linköping, Örebro and Uppsala, as well as the Karolinska University Hospital in Stockholm, Sweden, were invited to participate in the study in 2017. Clinical parameters related to diagnosis, including autoantibody status, were collected through patient chart review. Control data for the same questions were collected within the Epidemiological Investigation of Rheumatoid Arthritis (EIRA) $\mathrm{I}^{29}$ and EIRA II $^{30}$ studies during 1996-2014, which are part of a population-based project comprising the Swedish population.

The index date was defined as the date of pSS diagnosis for cases and the date of responding to the questionnaire for controls. A maximum of $n=15$ unique controls were matched to each pSS case based on age at index date, calendar time at index date, sex and area of residency. In analyses stratified by specific HLA haplotypes, controls were additionally required to have at least one allele (ie, heterozygotic) of the haplotype of interest.

The studies were approved by the Regional Ethics Committee in Stockholm and all participants gave informed consent to participate.

\section{Data collection}

Information regarding historical and current lifestyle habits was collected through standardised questionnaires, including questions on smoking where respondents were requested to provide the years and amount of smoking (online supplemental table 1). The response rates were $74 \%$ for cases in GESS, $80 \%$ for controls in EIRA I and $70 \%$ for controls in EIRA II. Missing data were excluded. For controls, HLA-DRB1 genotypes were determined as previously described. ${ }^{31}$ Cases were genotyped using the Illumina OmniExpressExome array, and HLA genotypes were imputed as previously described using tag SNPs and reference genomes. ${ }^{32}$

\section{Definition of smoking exposure}

Smoking exposure was defined as reporting having smoked on a regular basis prior to the index date. Smoking pack-years were estimated by multiplying the reported average smoking on a weekly basis by the length of a stated smoking period. Sensitivity tests including both records of regular and intermittent smoking were performed, in which the results were largely unaltered, and are therefore not included in the final analyses. Moreover, to account for potential reversed causality in instances where early symptoms of pSS might influence the likelihood of smoking, various latency periods were applied for smoking exposure that is only accounting for smoking in cases and controls occurring prior to the latency period.

\section{Statistical analysis}

ORs and 95\% CIs of smoking exposure were estimated using logistic regressions conditioned on the matching strata. Never-smokers were used as reference group. In analyses comparing the likelihood of stopping current smoking, ever-smokers in respective time-band were used as reference group. Interaction effects between smoking exposure and HLA haplotypes, assessed on a multiplicative scale, were also estimated.

All analyses were performed using STATA/MP version 13.0 (StataCorp LP, College Station, TX, USA). Statistical significance was defined by an alpha level of 0.05 . 


\section{RESULTS}

\section{Study population}

Of the patients with pSS returning the questionnaire $(\mathrm{n}=606), 530$ patients were matched to 4425 controls, and thus eligible for inclusion in further analysis. The median age at pSS diagnosis was 54 years (IQR: $43-61$ years); $93 \%$ of the cases were female and $71 \%$ were Ro/SSA and/or La/SSB autoantibody positive. The median time between pSS diagnosis and year of responding the questionnaire was 10 years. The pSS individuals included for analysis were similar to the entire group of patients with pSS responding to the questionnaire (table 1$)$.

\section{Smoker status at index date}

Thirty-seven per cent of the patients with pSS reported having ever smoked prior to pSS diagnosis date. Of the controls, $44 \%$ reported having ever smoked, resulting in

Table 1 Demographics of cases and controls

\begin{tabular}{|c|c|c|c|c|}
\hline & $\begin{array}{l}\text { pSS cases invited to } \\
\text { questionnaire }\end{array}$ & $\begin{array}{l}\text { pSS cases returning } \\
\text { the questionnaire }\end{array}$ & $\begin{array}{l}\text { pSS cases returning } \\
\text { the questionnaire and } \\
\text { matched to controls }\end{array}$ & Matched controls \\
\hline No. of individuals & 815 & 606 & 530 & 4425 \\
\hline$\%$ Females & 93 & 93 & 93 & 89 \\
\hline \multicolumn{5}{|c|}{ Age at index date (years) } \\
\hline Median (IQR) & $53(42-61)$ & $54(41-61)$ & $54(43-61)$ & $54(42-62)$ \\
\hline$\leq 24$ & $25(3 \%)$ & $14(2 \%)$ & $13(2 \%)$ & $117(3 \%)$ \\
\hline $25-39$ & $137(17 \%)$ & 114 (19\%) & $93(18 \%)$ & 795 (18\%) \\
\hline $40-54$ & $272(33 \%)$ & 197 (33\%) & $169(32 \%)$ & $1360(31 \%)$ \\
\hline $55-64$ & $222(27 \%)$ & $184(30 \%)$ & 173 (33\%) & 1437 (32\%) \\
\hline$\geq 65$ & $137(17 \%)$ & $95(16 \%)$ & $82(15 \%)$ & $716(16 \%)$ \\
\hline \multicolumn{5}{|c|}{ Calendar year at index date } \\
\hline $1980-1985$ & $7(1 \%)$ & $6(1 \%)$ & $0(0 \%)$ & $0(0 \%)$ \\
\hline $1985-1990$ & $37(5 \%)$ & $23(4 \%)$ & $0(0 \%)$ & $0(0 \%)$ \\
\hline $1990-1995$ & $59(7 \%)$ & $42(7 \%)$ & $13(2 \%)$ & $0(0 \%)$ \\
\hline $1995-2000$ & $131(16 \%)$ & 97 (16\%) & $94(18 \%)$ & $506(11 \%)$ \\
\hline 2000-2005 & $144(18 \%)$ & $112(18 \%)$ & $110(21 \%)$ & $857(19 \%)$ \\
\hline 2005-2010 & $196(24 \%)$ & $161(27 \%)$ & $156(29 \%)$ & $1809(41 \%)$ \\
\hline 2010-2015 & $214(26 \%)$ & $159(26 \%)$ & $154(29 \%)$ & $1253(28 \%)$ \\
\hline 2015-2020 & $3(0 \%)$ & $3(0 \%)$ & $3(1 \%)$ & $0(0 \%)$ \\
\hline \multicolumn{5}{|l|}{ Area of residence } \\
\hline $\begin{array}{l}\text { Bigger cities and } \\
\text { Southern Sweden }\end{array}$ & $609(75 \%)$ & $452(75 \%)$ & $406(77 \%)$ & 3763 (85\%) \\
\hline Middle Sweden & $194(24 \%)$ & $143(24 \%)$ & $115(22 \%)$ & $583(13 \%)$ \\
\hline Northern Sweden & $12(1 \%)$ & $11(2 \%)$ & $9(2 \%)$ & $79(2 \%)$ \\
\hline \multicolumn{5}{|c|}{ Highest attained education } \\
\hline $\begin{array}{l}\text { Primary education } \\
\text { ( } \leq 9 \text { years) }\end{array}$ & - & $134(22 \%)$ & $114(22 \%)$ & $1053(24 \%)$ \\
\hline $\begin{array}{l}\text { Secondary educatio } \\
\text { (10- } 12 \text { years) }\end{array}$ & - & $193(32 \%)$ & $172(32 \%)$ & $1601(36 \%)$ \\
\hline Higher education & - & $274(45 \%)$ & $241(45 \%)$ & $1764(40 \%)$ \\
\hline \multicolumn{5}{|c|}{ SSA and SSB status at the date of pSS diagnosis } \\
\hline SSA positive & $563(69 \%)$ & $418(69 \%)$ & $363(68 \%)$ & - \\
\hline SSB positive & $367(45 \%)$ & $269(44 \%)$ & $230(43 \%)$ & - \\
\hline $\begin{array}{l}\text { SSA and/or SSB } \\
\text { positive }\end{array}$ & $578(71 \%)$ & $430(71 \%)$ & $375(71 \%)$ & - \\
\hline \multicolumn{5}{|c|}{ No. of years between pSS diagnosis and questionnaire response date } \\
\hline Median (IQR) & $12(7-19)$ & $11(7-18)$ & $10(7-16)$ & - \\
\hline $\begin{array}{l}\text { No. of individuals with } \\
\text { HLA data }\end{array}$ & - & 409 (67\%) & $363(68 \%)$ & 2097 (47\%) \\
\hline
\end{tabular}

HLA, human leucocyte antigen; pSS, primary Sjögren's syndrome; SSA, Ro/SSA autoantibodies; SSB, La/SSB autoantibodies. 
an OR of 0.67 (95\% CI 0.55 to 0.81 ) for ever-smoking. An even lower OR was observed for current smoking at the pSS diagnosis date (OR $0.37,95 \%$ CI 0.26 to 0.53 ). Stratification of patients with pSS by Ro/SSA and La/SSB status resulted in similar ORs (table 2).

\section{Period prevalence of smoking prior to pSS diagnosis}

Next, to more thoroughly investigate smoking patterns preceding pSS diagnosis, period prevalence of smoking during the five decades preceding pSS diagnosis date was assessed separately. The fraction of ever-smokers during 49-40 years prior to pSS diagnosis date was not significantly different between cases and controls (OR: 0.89, $95 \%$ CI 0.66 to 1.20 ). However, the corresponding ORs for the following periods were consistently lower $(\mathrm{p}<0.05)$.
Moreover, individuals who developed pSS were more likely to discontinue smoking compared to controls (figure 1).

Stratification with regard to Ro/SSA and La/SSB status revealed a similar pattern for patients with and without autoantibodies, with ORs of smoking prevalence decreasing with greater temporal proximity to the pSS diagnosis date. Notably, patients with pSS without Ro/SSA and La/ SSB autoantibodies presented with the highest ORs for discontinuing smoking and with lower ORs for period prevalence of smoking during the three decades preceding diagnosis (figure 1).

Smoking exposure prior to pSS diagnosis was also stratified by age. Although largely not statistically significant, the ORs of smoking decreased with higher age (online supplemental table 2).

Table 2 Ever-, former-, current regular smoking and never-smoking at the time of pSS diagnosis date*

\begin{tabular}{|c|c|c|c|c|c|}
\hline \multirow[b]{2}{*}{ Patients with pSS } & \multirow[b]{2}{*}{ Exposure } & \multicolumn{2}{|c|}{ Risk estimate } & \multicolumn{2}{|c|}{$\mathrm{N}$ exposed $(\%) \dagger$} \\
\hline & & OR & $95 \% \mathrm{Cl}$ & Cases & Controls \\
\hline \multirow[t]{4}{*}{ All patients with pSS } & Ever-smoker & 0.67 & (0.55 to 0.81$)$ & $194(37)$ & $1961(44)$ \\
\hline & Former smoker & 0.81 & (0.65 to 1.00$)$ & $154(29)$ & $1244(28)$ \\
\hline & Current smoker & 0.37 & (0.26 to 0.53$)$ & $38(7)$ & 717 (16) \\
\hline & Never-smoker & Ref. & & $301(57)$ & $2027(46)$ \\
\hline \multirow[t]{4}{*}{ SSA- and/or SSB-positive pSS } & Ever-smoker & 0.70 & (0.55 to 0.89$)$ & $141(38)$ & $1365(44)$ \\
\hline & Former smoker & 0.82 & (0.63 to 1.06$)$ & $109(29)$ & $865(28)$ \\
\hline & Current smoker & 0.41 & (0.27 to 0.62$)$ & $30(8)$ & $500(16)$ \\
\hline & Never-smoker & Ref. & & $208(55)$ & $1429(46)$ \\
\hline \multirow[t]{4}{*}{ SSA-/SSB-negative pSS } & Ever-smoker & 0.58 & (0.40 to 0.85$)$ & $52(35)$ & $582(45)$ \\
\hline & Former smoker & 0.77 & (0.51 to 1.17$)$ & $44(29)$ & $370(29)$ \\
\hline & Current smoker & 0.28 & (0.13 to 0.59$)$ & $8(5)$ & $212(16)$ \\
\hline & Never-smoker & Ref. & & $90(60)$ & $577(45)$ \\
\hline
\end{tabular}

*Intermittent smoking was not included in exposure variables; never-smokers were defined as individuals reporting having never smoked regularly or intermittently.

tSums of former and current smokers lower than number of ever-smokers relate to missing data/inconsistent reporting of respondents. pSS, primary Sjögren's syndrome; SSA, Ro/SSA autoantibodies; SSB, La/SSB autoantibodies; Ref., reference.
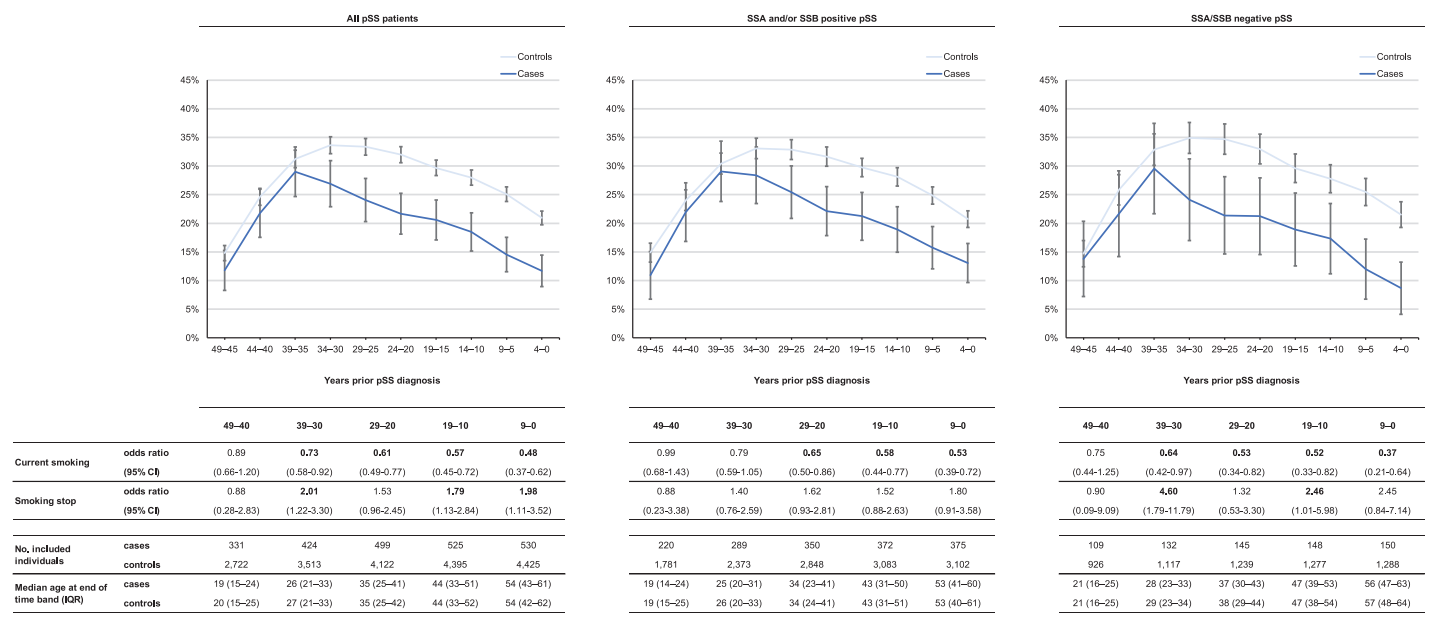

Figure 1 Period prevalence of smoking in relation to pSS diagnosis, stratified by SSA and SSB status. Error bars mark 95\% Cls. pSS, primary Sjögren's syndrome; SSA, Ro/SSA autoantibodies; SSB, La/SSB autoantibodies. 
Cumulative cigarette smoking pack-years prior to pSS diagnosis

To examine the potential dose-response relationships of smoking and pSS, ORs for reaching cumulative thresholds of smoking pack-years were calculated. The fraction of patients with pSS having cumulatively smoked $\geq 2$, as well as 4, pack-years at pSS diagnosis was significantly lower compared to the control group. As latency periods were applied, ORs increased closer to 1. A similar pattern was observed both in Ro/SSA- and/or La/SSB-positive and negative patients with pSS (figure 2). The aforementioned analyses were also performed for $\geq 10$ or $\geq 20$ pack-years, in which estimates were similar to the disclosed data.

\section{Smoking patterns and HLA haplotype in relation to the risk of developing pSS}

HLA profiles have been demonstrated to interact with smoking to increase the risk of developing several chronic inflammatory diseases, ${ }^{16} 17$ and were therefore incorporated in the analyses as a last step. In our cohort, HLA DRB1*03 and DRB1*15 genotypes were significantly enriched in patients with pSS with Ro/SSA and/or La/ SSB autoantibodies, whereas HLA-DRB $1 * 01, * 04$ and $* 10$ were less frequent (online supplemental tables 3 and 4 ). In patients without Ro/SSA and $\mathrm{La} / \mathrm{SSB}$ antibodies, none of the investigated HLA haplotypes were significantly more or less frequent than in controls.

Stratified by HLA haplotypes, current smoking at pSS diagnosis date was significantly less prevalent in all assessed groups. However, ever-smoking was only significantly less common in patients with pSS with HLA$\mathrm{DRB} 1 * 03$, although with $\mathrm{OR}<1$ in patients with pSS with HLA-DRB $1 * 15$ and HLA-DRB $1 * 01 / 04 / 10$. Interestingly, the lowest prevalence and OR of current smoking at pSS diagnosis was observed in patients with HLA-DRB1*01
/04/10 (table 3). Analyses of cumulative smoking packyears prior to pSS diagnosis resulted in relatively similar estimates across the different groups. However, no estimates indicated a significantly lower cumulative dose of smoking in patients with pSS with HLA-DRB1*15 compared to controls (figure 3). Interaction effects of smoking exposure and HLA haplotypes were assessed in a multivariate logistic regression model, in which no significant modifying properties from smoking and HLA combinations were observed (figure 4).

\section{DISCUSSION}

In this case-control study encompassing clinically validated pSS cases and matched controls, we observed a lower frequency of smoking preceding pSS diagnosis compared with the population at large. Investigations of smoking patterns over time revealed that individuals who later develop pSS smoke as much as the general population early in life, but were then significantly more prone to discontinue smoking. Strikingly, this behavioural change was observed as early as three to four decades prior to diagnosis. Our study is the first to provide a detailed description of smoking patterns preceding pSS diagnosis, which may be of key importance to understanding the role of smoking in pSS, and partly confirms previous studies showing lower frequencies of current smoking among patients with pSS. ${ }^{23} 2633$

A main finding of our study was the shift in smoking habits occurring approximately 30 years prior to diagnosis, a time during which the frequency of smoking markedly decreased among individuals later to be diagnosed with pSS relative to controls. This pattern can be explained by pSS cases being significantly more prone to stop smoking and/or not start smoking. Notably, this

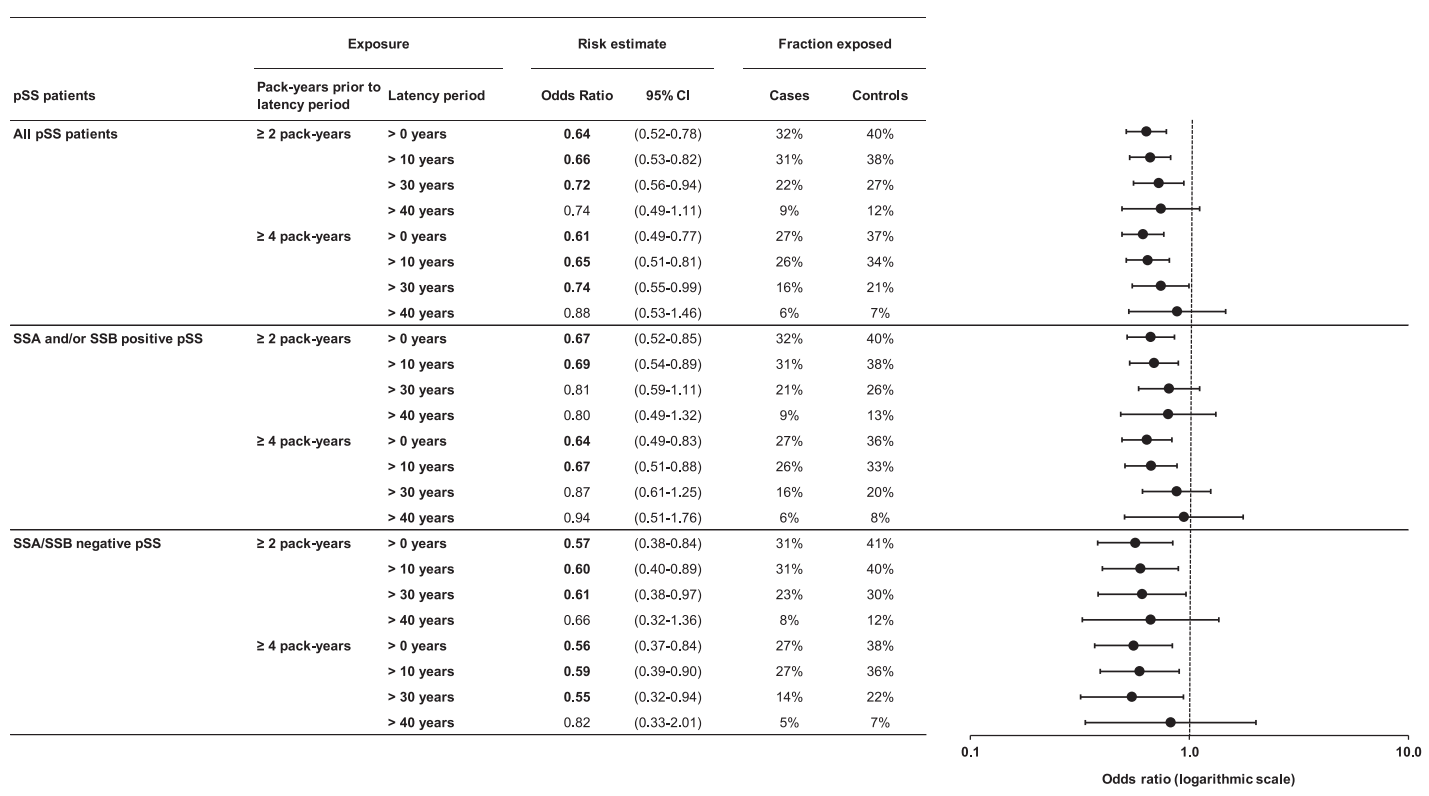

Figure 2 Cumulative pack-years of regular smoking prior to pSS diagnosis date, stratified by SSA and SSB status. Error bars demark 95\% Cls. pSS, primary Sjögren's syndrome; SSA, Ro/SSA autoantibodies; SSB, La/SSB autoantibodies. 
Table 3 Ever-, former-, current regular smoking and never-smoking at the time of pSS diagnosis date, stratified by HLA haplotype*

\begin{tabular}{|c|c|c|c|c|c|}
\hline \multirow[b]{2}{*}{ HLA haplotype (present in cases and controls) } & \multirow[b]{2}{*}{ Exposure } & \multicolumn{2}{|c|}{ Risk estimate } & \multicolumn{2}{|c|}{$\mathrm{N}$ exposed (\%)ף } \\
\hline & & OR & $95 \% \mathrm{Cl}$ & Cases & Controls \\
\hline \multirow[t]{4}{*}{ HLA-DRB1*03† } & Ever-smoker & 0.60 & (0.40 to 0.92$)$ & $55(35)$ & $240(47)$ \\
\hline & Former smoker & 0.72 & (0.46 to 1.13$)$ & $43(28)$ & $169(33)$ \\
\hline & Current smoker & 0.42 & (0.18 to 0.99 ) & $11(7)$ & $71(14)$ \\
\hline & Never-smoker & Ref. & & $89(57)$ & $219(43)$ \\
\hline \multirow[t]{4}{*}{ HLA-DRB1*15 } & Ever-smoker & 0.87 & (0.57 to 1.31$)$ & $58(44)$ & $254(46)$ \\
\hline & Former smoker & 0.97 & (0.62 to 1.51$)$ & $45(34)$ & $169(31)$ \\
\hline & Current smoker & 0.41 & (0.19 to 0.91$)$ & $12(9)$ & $85(15)$ \\
\hline & Never-smoker & Ref. & & $68(51)$ & $242(44)$ \\
\hline \multirow[t]{4}{*}{ HLA-DRB1*01/04/10§ } & Ever-smoker & 0.70 & (0.46 to 1.06$)$ & $46(39)$ & $376(48)$ \\
\hline & Former smoker & 0.95 & (0.60 to 1.50$)$ & 39 (33) & $244(31)$ \\
\hline & Current smoker & 0.27 & (0.11 to 0.66$)$ & $6(5)$ & $132(17)$ \\
\hline & Never-smoker & Ref. & & $67(56)$ & $339(43)$ \\
\hline
\end{tabular}

*Intermittent smoking was not included in exposure variables; never-smokers were defined as individuals reporting having never smoked regularly or intermittently.

$\dagger \mathrm{n}=155$ cases, $\mathrm{n}=515$ controls.

$\neq n=133$ cases, $n=554$ controls.

$\S n=119$ cases, $n=782$ controls.

ISums of former and current smokers lower than number of ever-smokers relate to missing data/inconsistent reporting of respondents.

HLA, human leucocyte antigen; pSS, primary Sjögren's syndrome; Ref., reference.

shift occurred prior to time points when self-reported symptom onset has been reported. ${ }^{19}$ While these data allow for different ways of interpretation, we suggest that these behavioural changes may potentially reflect very early, mild disease symptoms and underlying pathological changes. Disease symptoms and the presence of disease-associated autoantibodies are known to precede diagnosis by many years. ${ }^{34} 35$ Indeed, signs of autoimmune processes initiated several decades before diagnosis was described in a study by Theander et al, in which autoantibodies could be detected up to 20 years before pSS diagnosis. ${ }^{35}$ We speculate that such early processes may be associated with unrecognised pathological changes of mucosal surfaces, which may in turn influence

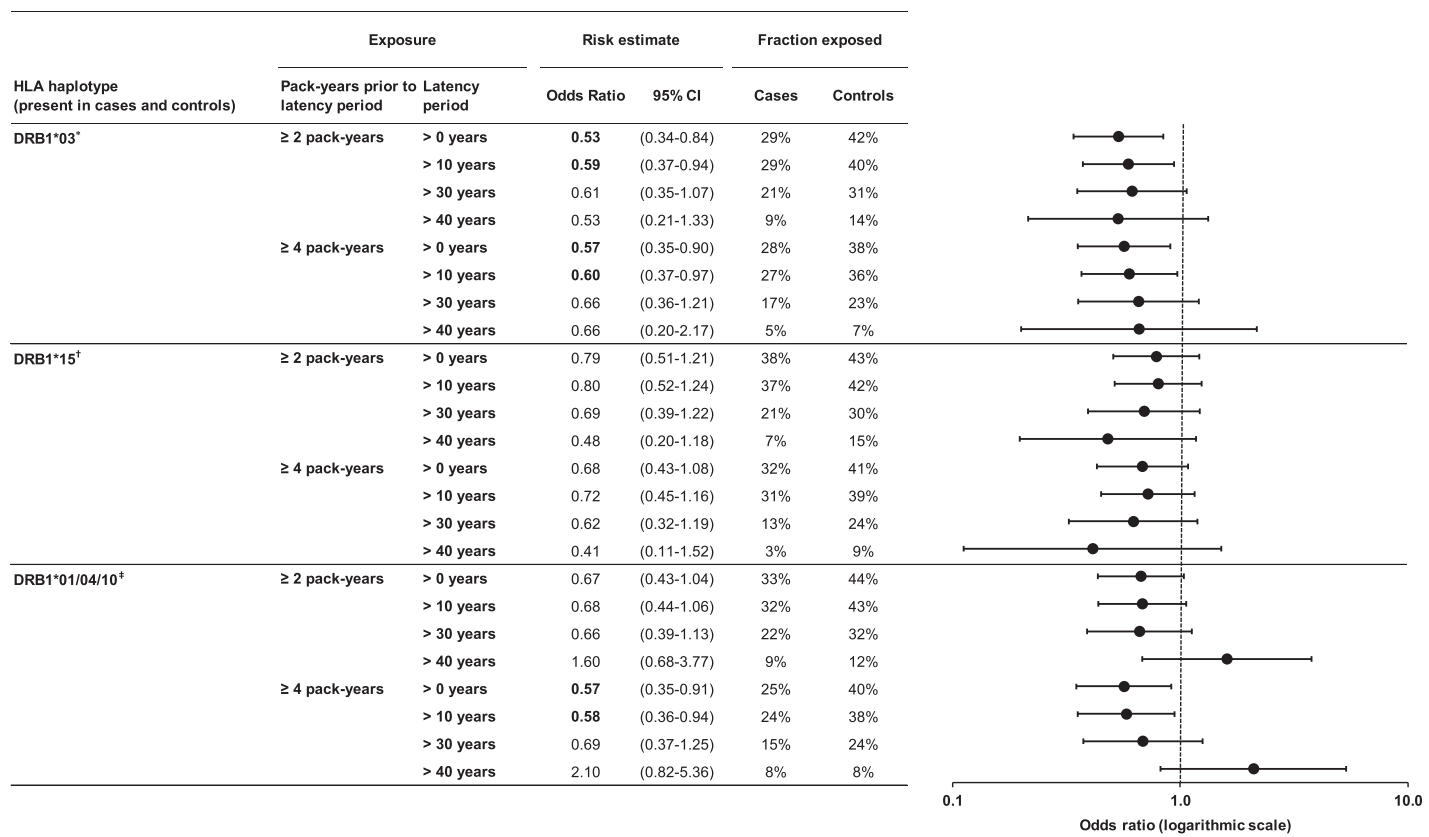

Figure 3 Cumulative pack-years of regular smoking prior to pSS diagnosis date, stratified by HLA haplotype. Error barks demark 95\% Cls. HLA, human leucocyte antigen; pSS, primary Sjögren's syndrome. ${ }^{*} n=155$ cases, $n=515$ controls; $† n=133$ cases, $\mathrm{n}=554$ controls; $\ddagger \mathrm{n}=119$ cases, $\mathrm{n}=782$ controls. 


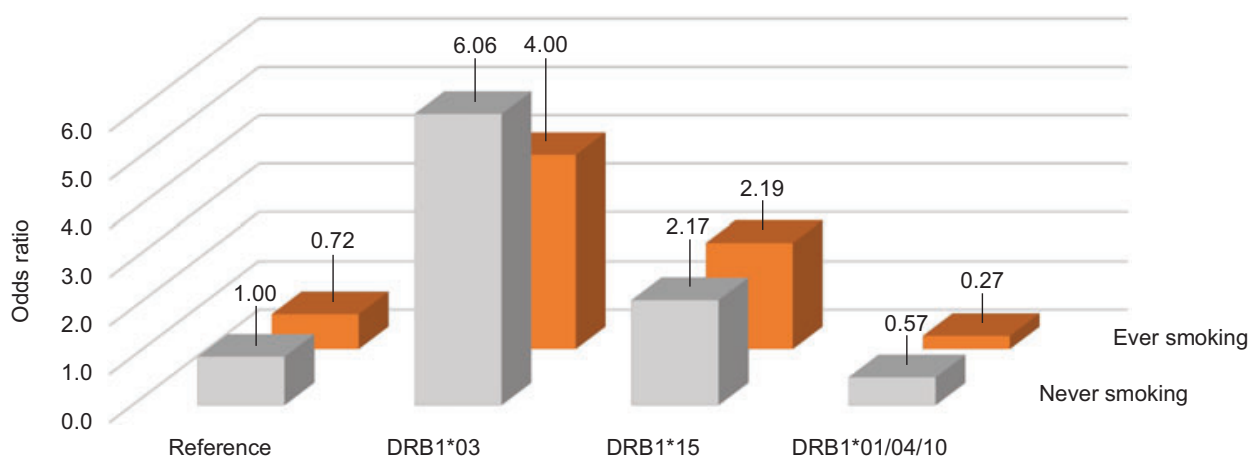

HLA haplotype

\begin{tabular}{|c|c|c|c|c|c|c|}
\hline \multirow[b]{2}{*}{ Exposure } & \multicolumn{2}{|c|}{ Never smoking } & \multicolumn{2}{|c|}{ Ever smoking } & \multicolumn{2}{|c|}{$\begin{array}{l}\text { Smoking \& HLA interaction } \\
\text { term }\end{array}$} \\
\hline & Odds Ratio & $95 \% \mathrm{Cl}$ & Odds Ratio & $95 \% \mathrm{Cl}$ & Odds Ratio & $95 \% \mathrm{Cl}$ \\
\hline Reference & & & 0.72 & $(0.34-1.50)$ & & \\
\hline DRB1*03 & 6.06 & $(3.89-9.45)$ & 4.00 & $(2.16-7.40)$ & 0.92 & $(0.46-1.81)$ \\
\hline DRB1*15 & 2.17 & $(1.35-3.48)$ & 2.19 & $(1.14-4.21)$ & 1.40 & $(0.70-2.83)$ \\
\hline DRB1*01/04/10 & 0.57 & $(0.35-3.48)$ & 0.27 & $(0.13-0.56)$ & 0.66 & $(0.31-1.38)$ \\
\hline
\end{tabular}

Figure 4 ORs of ever regular smoking and interaction effects with HLA-DRB1 alleles in patients with pSS with Ro/SSA and/or La/ SSB autoantibodies compared to controls. The reference group consists of never-smokers and non-carriers of the investigated HLAs; HLA, human leucocyte antigen; pSS, primary Sjögren's syndrome.

the likelihood to continue smoking. However, subclinical signs of disease multiple decennia prior to diagnosis have not been described in the literature thus far.

An alternative interpretation of our data could be that smoking mechanistically acts to decrease the risk of pSS. However, current smoking has been associated with a higher risk of the immunologically related diseases systemic lupus erythematosus and RA, ${ }^{29}{ }^{36}$ perhaps making it less likely that it would have a protective effect in pSS and further supporting the interpretation of a behavioural change caused by early symptoms of pSS rather than a biologically protective effect of cigarette smoke.

Knowledge on the role of smoking in pSS is scarce, and most previous studies on smoking have been performed in prevalent cases, not specifically studying exposures prior to diagnosis. ${ }^{23-26}$ However, consistent with our results, lower frequency of current smokers among prevalent pSS cases has been observed in several smaller studies. ${ }^{23} 2633$ Indeed, this observation has often been attributed to the fact that tobacco smoke may cause discomfort for patients due to dryness in the mouth, respiratory system and the eyes. Smoking habits prior to diagnosis were previously investigated in only one study by Olsson $e t a l,{ }^{27}$ where prediagnostic data from a health survey conducted years before diagnosis in $n=63$ patients with pSS were used. Similar to our observations, individuals who later developed pSS were less likely to be current smokers but more likely to be former smokers, giving further support to the validity of this observation.

Stratification by the presence of Ro/SSA and/or La/ SSB antibodies revealed similar patterns of smoking preceding pSS diagnosis in our study. Given the apparent differences in genetic risk variants, age distribution and clinical disease course comparing autoantibody-positive and -negative patients, ${ }^{5} 83738$ more pronounced differences between these two groups might have been anticipated. However, the observation that both subgroups stopped smoking at a higher degree than controls, that is, regardless if they developed Ro/SSA and/or La/SSB antibodies, further strengthens the hypothesis that early symptoms of pSS may cause this trend.

Smoking was also analysed in the context of riskassociated HLA carriage. The investigated HLAs were selected on the basis of previous studies showing that HLA-DRB $1 * 03$ is a risk factor for pSS and for the production of anti-Ro/SSA and anti-La/SSB autoantibodies, ${ }^{5} \quad 14 \quad 39$ while HLA-DRB $1 * 15$ is 
associated with production of anti-Ro/SSA autoantibodies in pSS and interacts with smoking to increase the risk of MS. ${ }^{5}{ }^{40}$ Also, HLA-DRB $1 * 01 / 04 / 10$ was included as a marker of shared epitope, which interacts with smoking to increase the risk of seropositive RA. ${ }^{31}$ Our data confirmed that HLA-DRB $1 * 03$ and HLA-DRB $1 * 15$ were significantly enriched only in autoantibody-positive patients. ${ }^{5}$ Regardless of HLA haplotype, current smoking was less frequent among cases than controls at the time of pSS diagnosis. Slight differences in smoking patterns were observed in different HLA strata, potentially reflecting that the relationship between smoking and pSS differ depending on the genetic context. However, risk-associated HLA alleles were not found to modulate the effects of smoking in an interaction analysis, which is in contrast to previous studies in RA and MS where gene-environment interactions between HLA and smoking have been identified. ${ }^{16}{ }^{17}$ As smoking did not emerge as risk factor for pSS, this is perhaps not surprising.

The study has some limitations to consider. First, being a questionnaire-based case-control study, it is inherently vulnerable to recall bias. However, the facts that the questionnaire included questions on many potential environmental factors and that no specific emphasis was put on smoking, as well as smoking at large being relatively less prevalent in pSS cases, decrease the risk of this possibility having a major influence on the results. On the other hand, the different definitions of the index date for cases and controls, with a median time between index date (pSS diagnosis) and questionnaire-response date of 10 years for cases, may lead to differences in recall bias. Further limitations include that the impact of passive smoking exposure was not accounted for and that misclassification of smoking may be increased longer back in time. However, these errors are presumably similar between cases and controls, thus not amplifying any observed associations. Lastly, although the participation rate was high, selective participation may cause bias. Yet, reassuringly, demographical and serological variables in the assessed group of patients with pSS were similar to the entire group of patients invited to the questionnaire.

Key strengths of the study relate to only including patients with pSS fulfiling internationally accepted criteria, $^{28}$ and the contextually large number of included cases. To the best of our knowledge, this is by far the largest study undertaken to investigate behavioural and environmental patterns preceding pSS. Moreover, we were able to stratify patients based on Ro/SSA and/or La/SSB autoantibodies, the presence of which demark a genetically and clinically distinct subgroup. ${ }^{5} 8$ Relatedly, the data enabled the assessment of combined effects from smoking and HLA genotype, which has previously not been investigated in pSS. Lastly, the frequency of autoantibodies and HLA haplotypes among the pSS cases mirrors that of previous studies to indicate that our material is indeed a valid representation of the pSS population as a whole.

\section{CONCLUSION}

In this case-control study, cumulative smoking exposure prior to pSS diagnosis was lower than compared to controls. However, observed smoking patterns indicate that individuals who later develop pSS smoke equally much as the general population in early life, but then are more prone to stop smoking. This shift occurs several decennia prior to the pSS diagnosis. We interpret the data to potentially reflect very early pathological changes, highlighting the slow, but progressive nature of pSS, and potential benefits from earlier diagnosis and treatment.

\section{Author affiliations}

${ }^{1}$ Division of Rheumatology, Department of Medicine Solna, Karolinska Institute, Karolinska University Hospital, Stockholm, Sweden

${ }^{2}$ Department of Public Health and Clinical Medicine, Rheumatology, Umeå University, Umeå, Sweden

${ }^{3}$ Department of Rheumatology, Faculty of Medicine and Health, Örebro University, Örebro, Sweden

${ }^{4}$ Department of Clinical Neuroscience, Karolinska Institute, Karolinska University Hospital, Stockholm, Sweden

${ }^{5}$ Division of Rheumatology, Department of Clinical Experimental Medicine, Linköping University, Linköping, Sweden

${ }^{6}$ Department of Clinical Sciences, Malmö, Rheumatology, Lund University, Malmö, Sweden

${ }^{7}$ Department of Medical Sciences, Rheumatology and Science for Life Laboratory, Uppsala University, Uppsala, Sweden

${ }^{8}$ Broegelmann Research Laboratory, Department of Clinical Science, University of Bergen, Bergen, Norway

Acknowledgements We thank Edith Ekström for excellent administrative support. Genotyping of cases was performed at the SNP\&SEQ Technology Platform, National Genomics Infrastructure (NGI), Science for Life Laboratory, Uppsala, Sweden.

Contributors $A B, J M, L A$ and MW-H conceived the study. AB, ERA and MW-H coordinated data collection from patient questionnaires. MK, HFdE, SM-B, PE, TM, GN and $A B$ recruited and characterised the patients. LP, IK and JH contributed with genotype and questionnaire data from controls. JM analysed the data with input from $\mathrm{LA}, \mathrm{MW}-\mathrm{H}$ and $\mathrm{AB}$. AB and JM drafted the first manuscript with input from $\mathrm{MW}-\mathrm{H}$ and LA. All authors participated in the editing of the manuscript until its final version.

Funding The study was supported by grants from the Swedish Research Council, the Swedish Rheumatism Association, the King Gustaf the V:th 80-year foundation, the Heart-Lung Foundation, the Freemason's in Stockholm Foundation for Children's Welfare, the Stockholm County Council, the Karolinska Institute, and the Torsten and Ragnar Söderberg Foundation. The SNP\&SEQ Technology Platform in Uppsala is supported by Science for Life Laboratory, the Swedish Research Council (VR-RFI), Uppsala University, and the Knut and Alice Wallenberg Foundation.

Competing interests None declared.

Patient consent for publication Not required.

Ethics approval The study was approved by the Regional Ethics Committee in Stockholm and all participants gave informed consent to participate.

Provenance and peer review Not commissioned; externally peer reviewed.

Data availability statement All data relevant to the study are included in the article or uploaded as supplemental information.

Supplemental material This content has been supplied by the author(s). It has not been vetted by BMJ Publishing Group Limited (BMJ) and may not have been peerreviewed. Any opinions or recommendations discussed are solely those of the author(s) and are not endorsed by BMJ. BMJ disclaims all liability and responsibility 
arising from any reliance placed on the content. Where the content includes any translated material, BMJ does not warrant the accuracy and reliability of the translations (including but not limited to local regulations, clinical guidelines, terminology, drug names and drug dosages), and is not responsible for any error and/or omissions arising from translation and adaptation or otherwise.

Open access This is an open access article distributed in accordance with the Creative Commons Attribution Non Commercial (CC BY-NC 4.0) license, which permits others to distribute, remix, adapt, build upon this work non-commercially, and license their derivative works on different terms, provided the original work is properly cited, appropriate credit is given, any changes made indicated, and the use is non-commercial. See: http://creativecommons.org/licenses/by-nc/4.0/.

ORCID iDs

Johannes Mofors http://orcid.org/0000-0003-1873-7169

Gunnel Nordmark http://orcid.org/0000-0002-3829-7431

Marie Wahren-Herlenius http://orcid.org/0000-0002-0915-7245

\section{REFERENCES}

1 Ambrosi A, Wahren-Herlenius M. Update on the immunobiology of Sjogren's syndrome. Curr Opin Rheumatol 2015;27:468-75.

2 Kvarnstrom M, Ottosson V, Nordmark B, et al. Incident cases of primary Sjogren's syndrome during a 5-year period in Stockholm County: a descriptive study of the patients and their characteristics. Scand $J$ Rheumatol 2015:44:135-42.

3 Mariette X, Criswell LA. Primary Sjogren's syndrome. N Engl J Med 2018;378:931-9.

4 Wahren-Herlenius M, Dorner T. Immunopathogenic mechanisms of systemic autoimmune disease. Lancet 2013;382:819-31.

5 Gottenberg JE, Busson M, Loiseau P, et al. In primary Sjogren's syndrome, HLA class II is associated exclusively with autoantibody production and spreading of the autoimmune response. Arthritis Rheum 2003;48:2240-5.

6 Halse AK, Marthinussen MC, Wahren-Herlenius M, et al. Isotype distribution of anti-Ro/SS-A and anti-La/SS-B antibodies in plasma and saliva of patients with Sjogren's syndrome. Scand J Rheumatol 2000;29:13-19.

7 Wahren-Herlenius M, Muller S, Isenberg D. Analysis of B-cell epitopes of the Ro/SS-A autoantigen. Immunol Today 1999;20:234-40.

8 Thorlacius GE, Hultin-Rosenberg L, Sandling J, et al. Genetic and clinical basis for two distinct subtypes of primary Sjögren's syndrome. Rheumatology (Oxford, England) 2020.

9 Bjork A, Mofors J, Wahren-Herlenius M. Environmental factors in the pathogenesis of primary Sjogren's syndrome. J Intern Med 2020;287:475-92.

10 Kuo CF, Grainge MJ, Valdes AM, et al. Familial risk of Sjogren's syndrome and co-aggregation of autoimmune diseases in affected families: a nationwide population study. Arthritis Rheumatol 2015;67:1904-12.

11 Aho K, Koskenvuo M, Tuominen J, et al. Occurrence of rheumatoid arthritis in a nationwide series of twins. J Rheumatol 1986;13:899-902. Available https://pubmed.ncbi.nlm.nih.gov/ 3820198/

12 Silman AJ, MacGregor AJ, Thomson W, et al. Twin concordance rates for rheumatoid arthritis: results from a nationwide study. $\mathrm{Br}$ J Rheumatol 1993;32:903-7.

13 Ulff-Moller CJ, Svendsen AJ, Viemose LN, et al. Concordance of autoimmune disease in a nationwide Danish systemic lupus erythematosus twin cohort. Semin Arthritis Rheum 2018;47:538-44.

14 Lessard CJ, Li H, Adrianto I, et al. Variants at multiple loci implicated in both innate and adaptive immune responses are associated with Sjogren's syndrome. Nat Genet 2013;45:1284-92.

15 Bolstad Al, Le Hellard S, Kristjansdottir G, et al. Association between genetic variants in the tumour necrosis factor/lymphotoxin alpha/ lymphotoxin beta locus and primary Sjogren's syndrome in Scandinavian samples. Ann Rheum Dis 2012;71:981-8.

16 Hedstrom AK, Katsoulis M, Hossjer O, et al. The interaction between smoking and HLA genes in multiple sclerosis: replication and refinement. Eur J Epidemiol 2017;32:909-19.

17 Klareskog L, Stolt P, Lundberg K, et al. A new model for an etiology of rheumatoid arthritis: smoking may trigger HLA-DR (shared epitope)-restricted immune reactions to autoantigens modified by citrullination. Arthritis Rheum 2006;54:38-46.
18 Igoe A, Scofield RH. Autoimmunity and infection in Sjogren's syndrome. Curr Opin Rheumatol 2013;25:480-7.

19 Mofors J, Arkema EV, Bjork A, et al. Infections increase the risk of developing Sjogren's syndrome. J Intern Med 2019;285:670-80.

20 Bjork A, Thorlacius GE, Mofors J, et al. Viral antigens elicit augmented immune responses in primary Sjogren's syndrome. Rheumatology (Oxford) 2019:1-11.

21 Klareskog L, Ronnelid J, Saevarsdottir S, et al. The importance of differences; on environment and its interactions with genes and immunity in the causation of rheumatoid arthritis. J Intern Med 2020;287:514-33

22 Olsson T, Barcellos LF, Alfredsson L. Interactions between genetic, lifestyle and environmental risk factors for multiple sclerosis. Nat Rev Neurol 2017;13:25-36.

23 Karabulut G, Kitapcioglu G, Inal V, et al. Cigarette smoking in primary Sjogren's syndrome: positive association only with ANA positivity. Mod Rheumatol 2011;21:602-7.

24 Manthorpe R, Benoni C, Jacobsson L, et al. Lower frequency of focal lip sialadenitis (focus score) in smoking patients. Can tobacco diminish the salivary gland involvement as judged by histological examination and anti-SSA/Ro and anti-SSB/La antibodies in Sjogren's syndrome? Ann Rheum Dis 2000;59:54-60.

25 Priori R, Medda E, Conti F, et al. Risk factors for Sjogren's syndrome: a case-control study. Clin Exp Rheumatol 2007;25:378-84. Available https://pubmed.ncbi.nlm.nih.gov/17631733/

26 Stone DU, Fife D, Brown M, et al. Effect of tobacco smoking on the clinical, histopathological, and serological manifestations of Sjogren's syndrome. PLoS One 2017;12:e0170249.

27 Olsson P, Turesson C, Mandl T, et al. Cigarette smoking and the risk of primary Sjogren's syndrome: a nested case control study. Arthritis Res Ther 2017;19.

28 Vitali C, Bombardieri S, Jonsson R, et al. Classification criteria for Sjogren's syndrome: a revised version of the European criteria proposed by the American-European Consensus Group. Ann Rheum Dis 2002;61:554-8.

29 Stolt P, Bengtsson C, Nordmark B, et al. Quantification of the influence of cigarette smoking on rheumatoid arthritis: results from a population based case-control study, using incident cases. Ann Rheum Dis 2003:62:835-41.

30 Hedstrom AK, Stawiarz L, Klareskog L, et al. Smoking and susceptibility to rheumatoid arthritis in a Swedish population-based case-control study. Eur J Epidemiol 2018;33:415-23.

31 Padyukov L, Silva C, Stolt P, et al. A gene-environment interaction between smoking and shared epitope genes in HLA-DR provides a high risk of seropositive rheumatoid arthritis. Arthritis Rheum 2004;50:3085-92.

32 Dilthey A, Leslie S, Moutsianas L, et al. Multi-population classical HLA type imputation. PLoS Comput Biol 2013;9:e1002877.

33 Juarez M, Toms TE, de Pablo $P$, et al. Cardiovascular risk factors in women with primary Sjogren's syndrome: United Kingdom primary Sjogren's syndrome registry results. Arthritis Care Res (Hoboken) 2014;66:757-64.

34 Jonsson R, Theander E, Sjostrom B, et al. Autoantibodies present before symptom onset in primary Sjogren syndrome. JAMA 2013;310:1854-5.

35 Theander E, Jonsson R, Sjostrom B, et al. Prediction of Sjogren's syndrome years before diagnosis and identification of patients with early onset and severe disease course by autoantibody profiling. Arthritis Rheumatol 2015;67:2427-36.

36 Takvorian SU, Merola JF, Costenbader KH. Cigarette smoking, alcohol consumption and risk of systemic lupus erythematosus. Lupus 2014;23:537-44.

37 Brito-Zeron P, Kostov B, Solans R, et al. Systemic activity and mortality in primary Sjogren syndrome: predicting survival using the EULAR-SS Disease Activity Index (ESSDAl) in 1045 patients. Ann Rheum Dis 2016:75:348-55.

38 Mofors J, Holmqvist M, Westermark L, et al. Concomitant Ro/SSA and $\mathrm{La} / \mathrm{SSB}$ antibodies are biomarkers for the risk of venous thromboembolism and cerebral infarction in primary Sjogren's syndrome. J Intern Med 2019;286:458-68.

39 Cruz-Tapias P, Rojas-Villarraga A, Maier-Moore S, et al. HLA and Sjogren's syndrome susceptibility. A meta-analysis of worldwide studies. Autoimmun Rev 2012;11:281-7.

40 Hedstrom AK, Sundqvist E, Baarnhielm M, et al. Smoking and two human leukocyte antigen genes interact to increase the risk for multiple sclerosis. Brain 2011;134:653-64. 